\title{
Problématique de l'Activité Cognitive des Jumeaux
}

\author{
F. Bak \\ Laboratoire de Psychologie Génétique Cognitive de Terrain, University of Lyon II
}

Résumé. Cette étude nous a permis de mettre à jour les différents modes d'intéractions qu'une mère établit avec ses jumeaux, ainsi que les évolutions possibles qui en résultent. Mais, bien plus, nous avons modélisé la façon dont les jumeaux élaborent leurs structures de l'activité de connaissance en fonction des sollicitations du milieu, ainsi que l'interdépendance qui les caractérisent.

Grâce à des examens opératoires, réalisés sur une population de 14 couples âgés de 6 a 10 ans, nous avons mis à jour un décalage intra-couple au niveau de la genèse de ces mêmes structures de l'activité de connaissance. Nous nous sommes apercus que sur le plan de la genèse de l'organisation cognitive l'un des enfants a développé des structures figuratives, et l'autre des structures opératives lui permettant d'avoir une connaissance de la réalité plus étendue.

Les liens de dépendance, de fusion, unissant les jumeaux entre eux, pourraient être dus à un développement cognitif parasitaire. Ils génèrent un lien gémellaire à partir de défaillances structurales qui vont apparaître chez chacun d'eux, ce qui ne permet aucunement leur autonomisation réciproque.

Puisque des déficiences apparaissent dans les structures de l'activité de connaissance de ces enfants, les problèmes qu'ils recontrent pourraient trouver leur solution dans une remédiation cognitive opératoire.

Termes clés: Interaction, Opérativité, Figurativité, Autonomisation, Parasitage

\section{INTRODUCTION}

Sans doute a-t-on et continue-t-on de considérer la gémellité comme un phénomène hors norme. La littérature, la bande dessinée, le cinéma se sont souvent emparés de ce thème qui fascine, interroge, effraie. Une multitude de travaux scientifiques ont aussi été réalisés depuis 1859 , date de la première trace d'une recherche utilisant des jumeaux. Si auparavant les jumeaux n'étaient considérés que comme des outils pour vérifier l'impact 
de la "nature" et de la "culture" chez l'homme, leur étude a fini par se recentrer sur eux-mêmes, à partir des années 30 . Et cela, jusqu'à les considérer comme deux individus ayant des relations différentes de toutes celles que l'on peut rencontrer habituellement $[29,30]$.

Trois grands axes de recherches se sont développés: il y a celles concernant les problèmes d'acquisition du langage, et la cryptophasie [4, 25]. Mais, parallèlement à ces recherches, des études portant sur la relation entre jumeaux d'un même couple sont apparues $[11,13,14,23]$. Enfin, un certain nombre de recherches concernant la relation de la mère aux jumeaux furent engagées suite aux travaux de Zazzo [1, 9, 21].

Notons pour information que c'est en 1951 que le terme de gémellologie fut créé par le professeur Luigi Gedda, également fondateur de la revue internationale: Acta Geneticae Medicae et Gemellologiae, Twin Research. Mais si la littérature concernant la gémellité est très florissante, les problèmes posés par les jumeaux sont loin d'être résolus, notamment ceux liés à leur autonomisation, à leur individuation.

Afin de bien mettre à jour la façon dont se génère cette problématique, il nous faut partir de la naissance des jumeaux, et les difficultés que peut rencontrer une mère qui essaie de considérer ses deux nourrissons de façon individuelle, en menant avec chacun une relation séparée $[21,22,24]$. Pour pallier les différents problèmes matériels qui se présentent il est fréquent que la mère réunisse ses enfants en une entité unique: l'entité gémellaire. Ce mode d'interaction se rencontre de façon plus marquée chez des jumeaux de même sexe [5]. En effet, ils bénéficient de soins qui sont en tous points identiques et pratiqués de façon similaire.

\section{Première période (0-2 ans)}

Ainsi, sur le plan de la genèse des structures cognitives, il semblerait que le développement qui va être amorcé est bien plus celui de l'entité gémellaire que celui de chacun des enfants dans leur singularité. Il y a donc un décalage au niveau de la structuration de la réalité, comparativement à un enfant unique, les jumeaux ayant une étape supplémentaire dans le développement de leur personne. Des travaux ont mis à jour un tel retard, confortent cette théorie [26, 27, 28]. Mais ceci implique que toutes les recherches visant à comparer des populations de jumeaux et de non jumeaux présentent un intérêt limité en ce qu'elles permettent seulement d'établir un décalage sans pouvoir l'expliquer.

Au cours des deux premières années de la vie, les premières procédures par lesquelles tout enfant prend connaissance du réel vont consister à le copier, à le décripter en fonction des systèmes de traitement dont il dispose. A ce niveau, c'est un contrôle externe qui est exercé par le milieu sur l'activité des schèmes. Mais progressivement, une fois que ce premier mode d'appréhension va être poussé à ses limites, l'enfant va le dépasser pour faire subir des transformations au réel. Progressivement va émerger un contrôle interne, une auto-régulation exercée par le sujet sur sa propre activité $[15,16]$. La dialectique générale de la structuration de la connaissance va donc être amorcée, bien que chez les jumeaux elle concerne, dans un premier temps, l'entité gémellaire. Le figuratif (qui est: “[...] la capacité de penser, ou encore d'agir en pensée sur des contenus présents, qu'ils soient perceptifs et/ou imagés, c'est à-dire sur des configurations perceptives ou imagées" [7] va progressivament céder la place à l'opératif (qui permet de raisonner sur 
des contenus réels ou évoqués par anticipation et rétroaction) qui se développe indépendamment mais en interaction avec lui.

Ce niveau d'opérativité va permettre à l'enfant d'envisager le monde sous une forme plus complexe que précédemment, d'avoir davantage d'emprise sur celui-ci et donc de générer une nouvelle forme de figurativité plus élaborée que la précédente. Cette alternance, se reproduisant sans cesse, permet aux procédures de prise de connaissance de la réalité d'être intégrées dans un système dont la complexité est croissante. L'évolution des structures de l'intelligence va donc résider dans le passage d'une adaptation restreinte, à une adaptation plus importante car elle englobe une plus grande partie de la réalité. Ainsi, les procédures figuratives et opératoires de la période sensori-motrice sont d'un niveau bien moins complexe que celles des périodes opératoire concrête et formelle.

Il convient de poser certaines limites quant au développement de l'entité gémellaire, chez tous les jumeaux. En effet, au regard de multiples recherches menées sur ce type de population, cette étape de l'entité gémellaire serait absente chez des jumeaux ayant eu:

- une prématurité importante (terme inférieur à 35 semaines)

- une hypotrophie importante (poids de naissance inférieur à 2,200 g)

- une présence de problèmes médicaux graves à la naissance

- une séparation prolongée de la mère.

Des couples de jumeaux appartenant à une telle population ne débutent pas leur développement cognitif par cette première étape (entité gémellaire) que nous venons de caractériser. Cette affirmation trouve sa justification dans des études ayant démontré que la mère, dont l'un de ses enfants possède les caractéristiques précédemment citées, va être plus procke de l'enfant le moins atteint sur le plan somatique [12]. Cette préférence va se poursuivre jusqu'aux environs de 3 mois et s'inverser par la suite.

Les conséquences d'une telle préférence d'un des jumeaux par rapport à l'autre vont entraîner d'importantes répercussions sur le plan cognitif. En effet, l'enfant sollicité commence à développer certains schèmes grâce à une interaction plus intense avec son entourage, alors que son jumeau est délaissé. Puis, il y a un retournement du mode d'interaction préférentielle (un tel mode de fonctionnement pouvant aller jusqu'au paroxysme de ne plus apporter que le strict minimum de soins, nécessaires à la survie de l'enfant). L'ensemble des acquisitions des enfants va donc se faire selon un mode qui correspondrait à notre seconde étape.

\section{Deuxième période (2 à 6-7 ans)}

Celle-ci est caractérisée par un développement cognitif parasitaire de l'un des jumeaux à l'égard de l'autre. L'un des enfants structurerait son activité de connaissance selon la dialectique du figuratif et de l'opératif précédemment posée; mais son frère, ou sa sœur suivant le cas, ne développerait que des procédures bien moins efficientes, parfois même totalement figuratives (ceci par un manque d'interactions, de réponses fournies face aux interrogations posées par le réel: l'attachement de la mère pour ses jumeaux va se faire en fonction de celui qu'elle imaginera être le plus dépendant de l'adulte et le moins compétent [1]). Cet enfant, "délaissé" devient donc plus sensible aux configurations des 
états qu'aux transformations les ayant produit ou les modifiant. Cette hypothèse est confortée par des travaux, qui établissent la présence d'un décalage entre jumeaux d'un même couple vis-à-vis de l'efficience verbale [4].

\section{Troisième période (à partir de 6-7 ans)}

C'est donc le milieu dans lequel évolue ces enfants qui sera le facteur déclenchant de ce processus de "parasitage". Or, c'est ce même milieu qui va pouvoir atténuer les répercussions d'un tel mode d'interaction. On a mis en évidence la présence d'une fluctuation dans ce mode de préférence au niveau de l'interaction mère/enfant $[6,9]$. Ainsi, chacun des jumeaux va à son tour se trouver placé au centre des interactions que le milieu établit avec le couple gémellaire. Les jumeaux vont donc avoir la possibilité de développer des structures plus efficientes sans s'emprisonner dans ce lien gémellaire qui attache le jumeau qui est dans la figurativité à celui qui est opératif. Une certaine forme d'autonomisation pourra donc être atteinte au cours de cette troisième étape.

Ce mode de structuration par "à coups" est un argument supplémentaire qui nous permet d'expliquer le retard qu'ont les couples de jumeaux dans leur développement. Bien que les structures qu'ils mettent en place leur permettent de s'adapter à la réalité, d'intégrer toute sa complexité, le temps qu'ils mettent à les construire est bien plus long que chez des enfants uniques.

Ces trois étapes conditionnent le développement cognitif des jumeaux mais ne se retrouvent pas uniquement dans une macro-genèse de l'élaboration des structures de l'activité de connaissance (dans une prévalence d'une étape en fonction de la période dans laquelle ils se situent). Nous pouvons aussi postuler qu'elles se retrouvent dans une micro-genèse de ce même développement (toutes les déséquilibrations et les rééquilibrations qui apparaîtront au sein du couple). Mais chaque nouvelle étape atteinte est influencée par celle qui lui est antérieure, car elle l'intègre dans une dynamique sans cesse croissante de l'évolution des connaissances. S'il y a une similitude dans les étapes qui se reproduisent, ce n'est qu'une similitude de forme, le contenu, lui, diffère.

\section{Représentation graphique}

L'ensemble des processus interactifs qui vont se succéder au cours du développement des jumeaux peut être représenté sous la forme d'un graphique où nous trouverions:

Sur l'axe des ordonnées, les trois étapes que nous posons comme étant les trois façons d'interagir avec les jumeaux:

- étape 1: unification en une entité

- étape 2: "complémentarité" des enfants

- étape 3: individuation des enfants

Sur l'axe des abscisses, les différents stades décrits par Piaget avec les âges qui les caractérisent:

- stade sensori-moteur (0 à 2 ans)

- stade pré-opératoire, ou symbolique ( 2 à 7 ans environ)

- stade opératoire concret (7-8 à 11-12 ans)

- stade formel (12 à 16 ans) 


\section{Période sensori-motrice}

Ainsi, au cours de la période sensori-motrice, les jumeaux dépendent totalement de leur environnement comme tout enfant, et ne peuvent survivre que grâce à celui-ci. Mais ce milieu, face à cette situation difficilement gérable, va les unifier en une entité. Il est en effet bien plus simple lorsque deux enfants pleurent de leur soumettre une seule réponse globale pour les apaiser, plutôt que de considérer chacun des enfants comme étant à la source de demandes différentes. L'étape 1 est donc celle qui va dominer au cours de toute cette période du développement [10].

Néanmoins, la prédominance de cette étape n'exclue pas l'apparition, momentanée certes, des étapes 2 et 3 car certains parents mettent en place des stratégies très complexes pour s'occuper de leurs jumeaux, basées sur l'alternance des soins qu'ils apportent à chacun d'eux. Cependant, cela ne perdure guère [21, 22]). Précisons cependant que les différents accès aux niveaux 1 et 3 n'offrent aucune indication de date car il ne s'agit que d'un schéma théorique. Nous aurions donc la représentation graphique suivante.

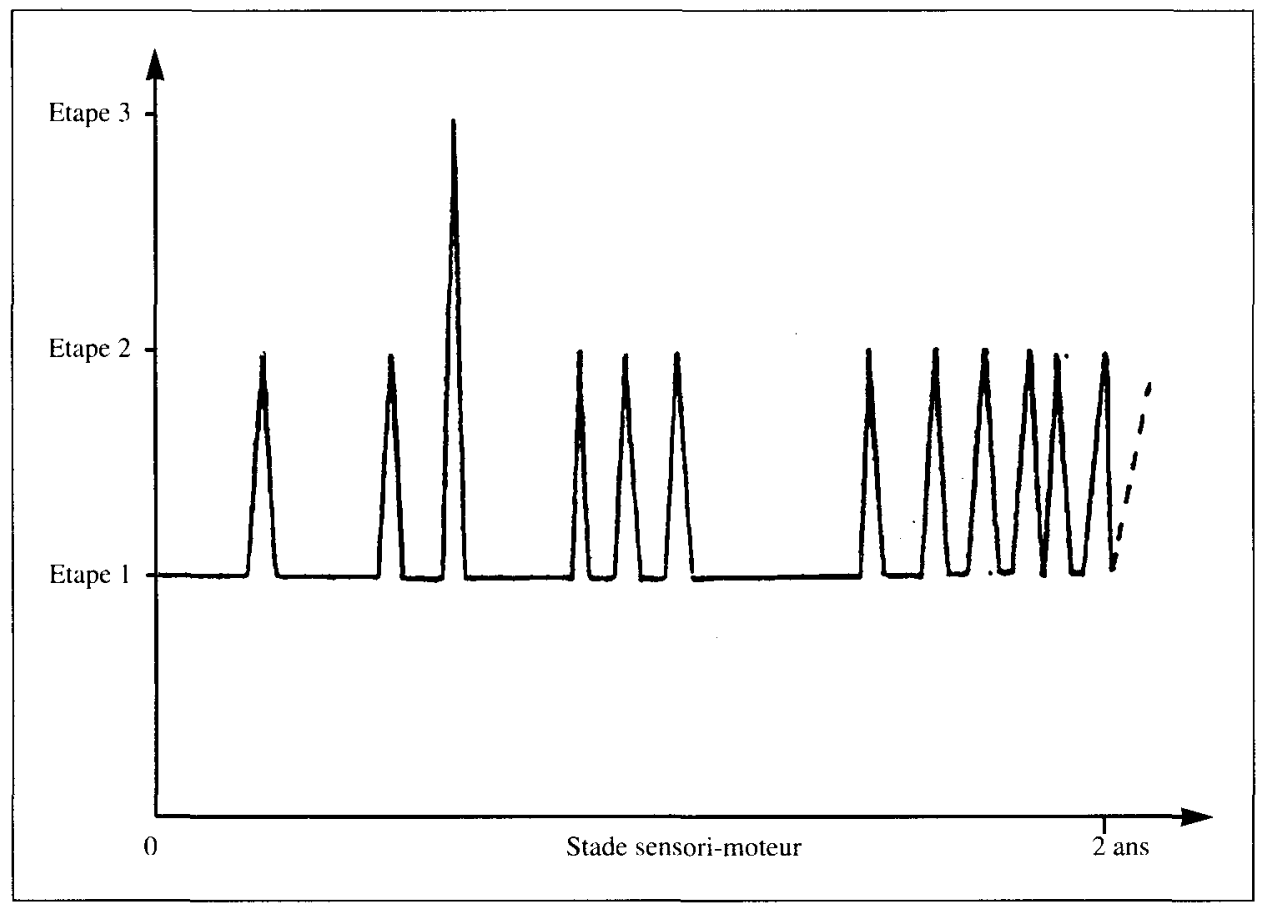

Schéma n. 1. Aspect pratique (unification)

\section{Stade pré-opératoire}

Puis, à la période pré-opératoire, les jumeaux accèderaient à l'étape de complémentarité (sur le plan extérieur), de "parasitage" (sur le plan interne de la genèse des structures cognitives). Ce mode d'interaction se mettrait en place à cause de la nécessité naissante 
de poser chaque enfant en tant qu'individu. Ceux-ci occupant de plus en plus l'espace qui les entoure.

Toutefois, ne pouvant passer d'un état d'unification à un mode d'interaction individualisant chacun des enfants, l'étape 2 serait présente en tant que transition vers l'étape 3. Or, puisque nous nous situons dans la genèse d'interactions influant sur les structures cognitives des jumeaux, nous allons trouver des oscillations qui tendent à un retour au premier mode de relation, avec l'entité gémellaire. Ces dernières vont devenir de plus en plus rares pour progressivement laisser place à des oscillations qui permettent cette fois une accession à l'étape 3 .

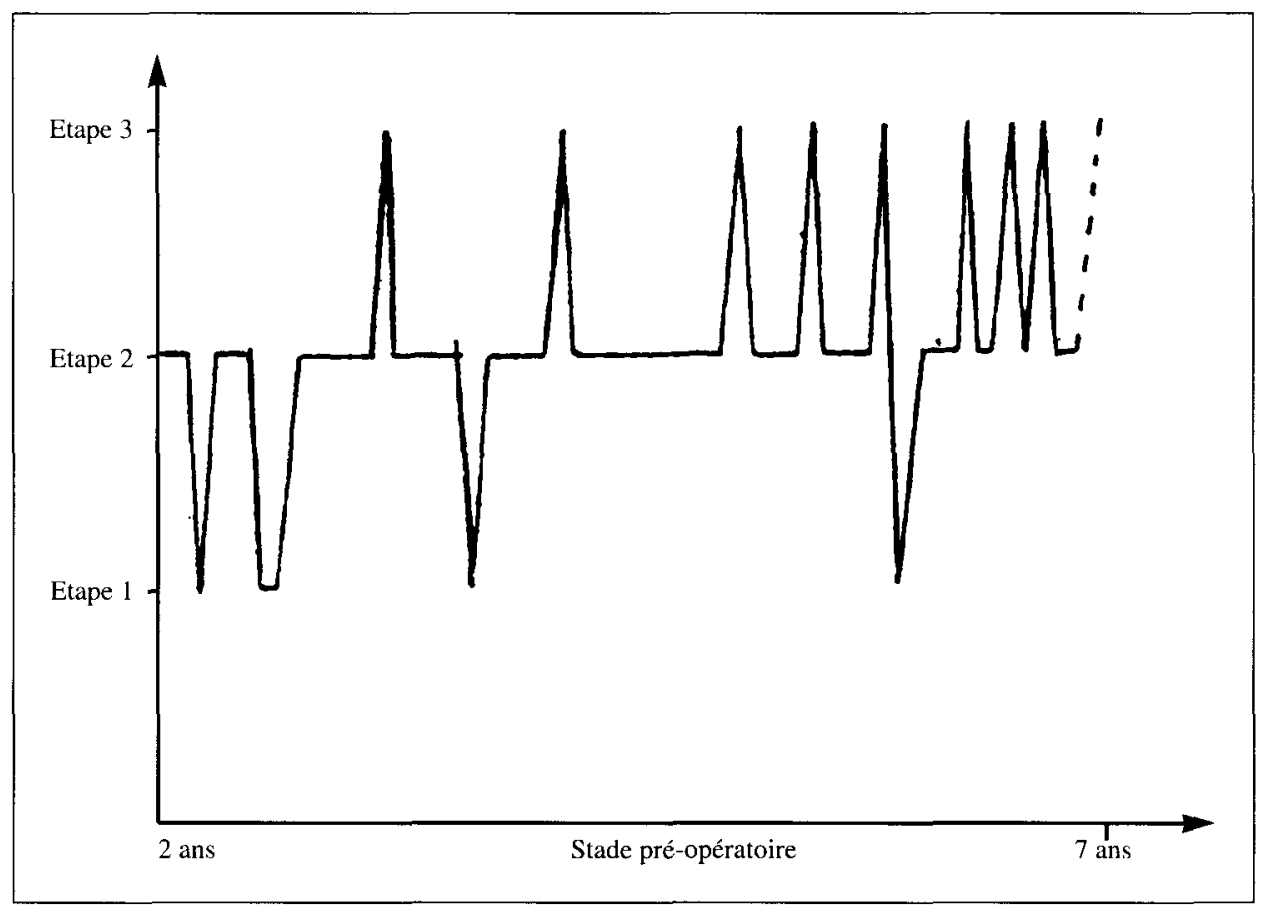

Schéma n. 2. Nécessité de les poser en tant qu'individus (= > prémices = complémentarité)

\section{Stade opératoire concret}

Enfin, nous en arrivons à la période opératoire concrète, où les enfants se stabiliseraient au niveau de l'étape 3, dite d'individuation. Par la scolarité qui leur est imposée, par l'autonomie de plus en plus grande qui est la leur vis-à-vis de leur milieu, les jumeaux vont être dorénavant considérés en tant qu'individus à part entière. Ainsi, progressivement un palier d'équilibre caractérisé par une autonomisation totale des enfants l'un à l'égard de l'autre va apparaître; cette étape se prolongeant au stade formel. 


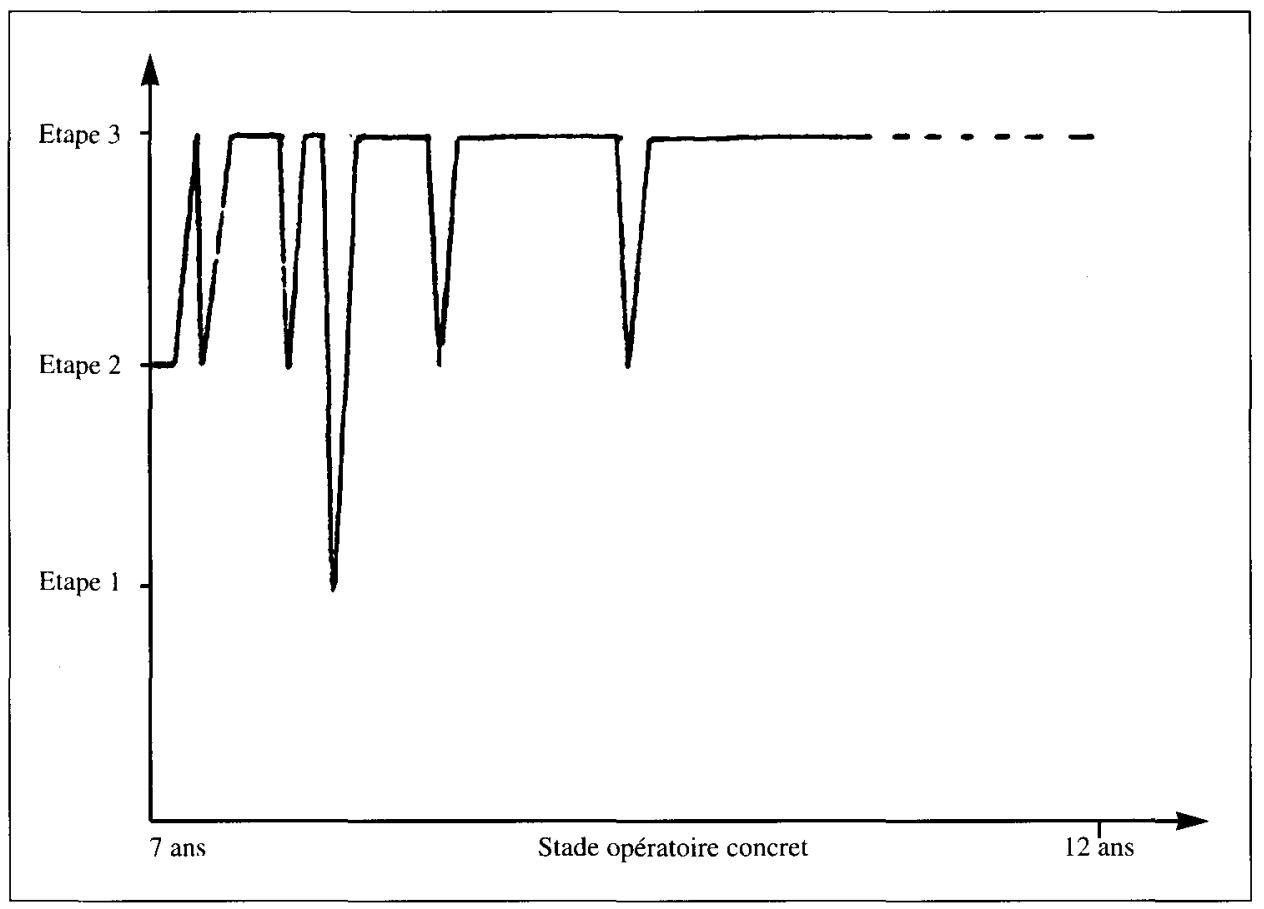

Schéma n. 3. Positionnement en tant qu'individu à part entière

L'ensemble de la genèse que nous venons d'évoquer permettrait d'aboutir à une parfaite individuation des jumeaux, se concluant par une maturation cognitive autonome, au stade formel. Mais, tous les couples de jumeaux n'ont pas un développement aussi théoriquement parfait. Une multitude d'entre eux a structuré la réalité selon des procédures que nous nommerons $\mathrm{B}$ ou $\mathrm{C}$, en référence à la désignation d'une individuation parfaite sous la lettre $A$.

$\mathrm{Au}$ niveau $\mathrm{B}$, nous trouverions des enfants qui, sortis de la période pré-opératoire, n'auraient pas atteint l'étape 3. Ceci étant dû à un milieu qui aurait favorisé un développement cognitif parasitaire. Le lien gémellaire, intense, qui unit ces enfants (empêchant leur autonomisation) et la dépendance présente sur le plan affectif trouverait donc sa source sur le plan cognitif, l'un rétro-agissant sur l'autre et réciproquement, par la suite. Leur structuration de l'activité de connaissance se développe donc en ne favorisant pas leur autonomisation.

Enfin, en C, nous trouverions des jumeaux qui n'auraient jamais dépassé l'unification totale qui est présente au stade sensori-moteur. A la période préopératoire, l'étape 2 de complémentarité ne se serait pas affirmée ou trop peu, n'ayant laissé aucune possibilité de passage à l'étape 3. Nous aurions donc des jumeaux qui auraient structuré la réalité selon des procédures peu élaborées, et quasiment identiques, ce qui les amèneraient par la suite à mener des vies similaires. Il n'en demeure pas moins que l'organisation de départ va toujours être modifiée, de façon progressive, mais avec un niveau d'efficience moindre que ce qu'il devrait être. 


\section{MATÉRIEL ET MÉTHODE}

\section{Sélection}

Pour vérifier notre théorie, concernant un décalage de la maturation des structures cognitives entre jumeaux d'une même paire qui influencerait leur autonomisation réciproque, nous avons réuni un groupe d'étude de 14 couples. L'ensemble de cette population a été choisie au sein d'un répertoire des naissances gémellaires, depuis 1970, que nous avons réalisé sur la ville de Lyon, à partir de critères très précis.

Tout d'abord, nous avons centré notre étude sur des enfants ayant entre 7 et 9 ans. C'est à cette période que se développe un mode de pensée qui émerge de la figurativité. La pensée opératoire va se présenter à travers l'émergence d'invariants liés à la substance, au poids, aux longueurs..., et dans l'organisation du monde en classes (avec tout ce qu'elles sous-tendent). Ainsi, il est possible de mettre à jour un décalage très net, quant au développement de cette pensée permettant à l'enfant d'avoir une plus grande emprise sur le réel, qui est dû à une trop grande prégnance de la figurativité. Nous avons néanmoins élargi notre population, pour 5 couples, à des enfants ayant aux alentours de 6 ans, pour examiner ce qu'il en était à l'entrée de la période pré-opératoire, et aux alentours de 10 ans pour étudier la fin du stade opératoire concret.

En outre, nous avons aussi restreint notre échantillonnage à des enfants n'ayant pas connu de problèmes, lors de leur naissance, tels que ceux décrit page 3 de notre exposé théorique (ces renseignements, très importants pour établir la possible participation de ces enfants à notre recherche, ont été obtenus à la suite de rencontres avec les parents). Nous ne reviendrons pas sur les raisons d'une telle délimitation, celles-ci ayant déjà été explicitées. Nous avons ainsi regroupé:

- 10 couples dizygotes (dont 2 entre 6-7 ans et 2 entre 9-10 ans)

- 4 couples monozygotes (dont 1 entre 10-11 ans).

Une fois notre liste de jumeaux définie, nous avons choisi de travailler avec eux au sein de l'établissement scolaire où ils se trouvaient pour avoir un cadre de travail suffisamment neutre.

L'ensemble de nos passations a été réparti en deux demi-journées pour chaque couple, avec une alternance des enfants. Nous avons aussi pris garde à ce que les enfants ne se rencontrent pas, au cours d'une même passation au moment de leur permutation, ceci pour éviter que les réponses données par l'un ne se transposent chez le second.

\section{L'examen opératoire}

La première étape pratique de notre étude a donc été de mettre en évidence, avec précision, le niveau de développement cognitif de ces jumeaux, et donc de découvrir les possibles défaillances existant dans leur genèse des structures logico-mathématiques et infralogiques. Nous avons donc employé la méthode de l'examen opératoire [8].

Cette méthode repose sur une discussion dirigée par le psychologue à propos d'une situation problème, celui-ci tentant de suivre l'enfant dans les méandres de sa pensée. “L'intérêt du diagnostic se situe donc dans l'analyse des mécanismes de la déduction 
reflétant par la même les possibilités ou les incapacités à effectuer les coordinations d'actions" [3]. L'ensemble du questionnement permet donc à l'enfant d'expliquer ce qu'il a fait, comment est-ce qu'il comprend les données du problème, les déductions l'ayant amené à la réponse qu'il a donnée, ce qui nous permet d'analyser les procédures qu'il utilise. Ces situations problèmes que nous proposons aux enfants proviennent des épreuves que Piaget a développées dans l'ensemble de son oeuvre $[17,18,19,20]$. Elles concernent plus particulièrement:

\section{le domaine infra-logique}

- les conservations physiques (substance, poids, volume)

- les conservations spatiales (longueurs, surfaces, la construction de l'horizontale, de la verticale...)

- les quantités continues

\section{le domaine logico-mathématique}

- les classes (quantification de l'inclusion, dichotomies, multiplication de critères, classifications hiérarchiques...)

- les quantités numériques (quantités discontinues, correspondance terme à terme, sériation...).

Il est à noter que la nature des épreuves proposées est relative au niveau de compétence des enfants, défini au cours de la passation. Ainsi, chaque examen est propre à chaque enfant ce qui nous permet de suivre au plus près son niveau de structuration. Si nous ne pouvons comparer de façon précise les couples entre eux, ce qui présente dans notre cas un intérêt réduit, il nous est cependant possible de mettre en parallèle les modalités de structuration de la réalité de chacun des enfants au sein d'un même couple.

Les seules règles invariantes que nous nous imposons sont d'une part de toujours alterner des épreuves appartenant à chacune des deux catégories de connaissance précédemment citéés, pour ne pas qu'il y ait de transfert d'apprentissage; et d'autre part de ne jamais nous satisfaire d'une réponse donnée par l'enfant, même si celle-ci semble correcte, mais de constamment chercher à le pousser dans ses justifications, dans l'exposition de sa logique.

\section{RÉSULTAT}

Suite à nos investigations, l'ensemble des protocoles que nous avons recueillis nous a permis de confirmer l'hypothèse que nous avions posée. Ainsi, dans l'ensemble des couples formant notre population, nous avons trouvé un des jumeaux en décalage vis-à-vis de l'autre (indépendamment du sexe des enfants, et de leur caractère monozygote ou dizygote; bien que nous ayons pu remarquer que chez les couples dizygotes mixtes la fille est constamment en avance sur son frère), sur le plan de la maturation des structures 
cognitives. Mais cet écart n'est en aucun cas stable, dans le sens où il n'est caractéristique que d'un lien gémellaire unissant une seule paire de jumeaux.

Démontrer cela ne vient que confirmer les propos tenus par Zazzo, qui affirme que “...s'il s'agit d'un couple de jumeaux, ces variations (du milieu à l'égard des enfants) ne sont pas exactement synchrones pour les deux partenaires" [29]. Ainsi, chaque enfant se développe à son propre rythme. Or, nous posons qu'un des enfants se trouve parasité dans son développement cognitif par son jumeau.

\section{Taxinomie}

En utilisant l'ensemble des variations que nous avons découvert, il nous est apparu qu'en répertoirant les réponses données par les enfants selon la taxinomie établie [8] que nous présenterons ensuite, nous pouvions calculer un degré d'autonomisation des jumeaux l'un à l'égard de l'autre, et par là même, quantifier l'intensité du lien gémellaire. Cette taxinomie classe les réponses des enfants selon une figurativité des plus importantes à une opérativité basée sur la réversibilité opératoire (capacité qu'a la pensée de revenir à un état initial, suite à une transformation, sans mise en acte dans le réel). Elle débute par des réponses:

\section{figuratives}

- perception d'un état, description de celui-ci par abstractions empiriques (conservation de la substance: “C'est pas la même chose de pâte, là c'est applati et là c'est gros"') - évocation de l'état initial face à l'état actuel (conservation de la substance: "C'est la même boule, avant elle était en boule"').

- évocation de la transformation ayant abouti à l'état initial (conservation de la substance: “Avant j'avais fait en boule, et j'ai mis pareil dans les deux")

- évocation des transformations ayant abouti à l'état final (conservation de la substance:

"J'ai cassé la boule seulement, alors ça fait toujours pareil.")

- transformation possible de l'état initial en état final "Y suffit de faire une galette pareille avec l'autre boule, on verra ça fait pareil."')

\section{operatives}

- retour possible de l'état final à l'état initial, au présent ("Si les morceaux on remet en boule, c'est pareil."')

- retour possible de l'état final à l'état initial, au conditionnel ("Si on les rassemblait, on verrait."').

nous avons comptabilisé les réponses des enfants, à chacune des épreuves infralogiques, dans chacune des catégories de la taxinomie. Les totaux obtenus sont additionnés puis traduits en pourcentages, selon la réorganisation suivante. 
Tableau

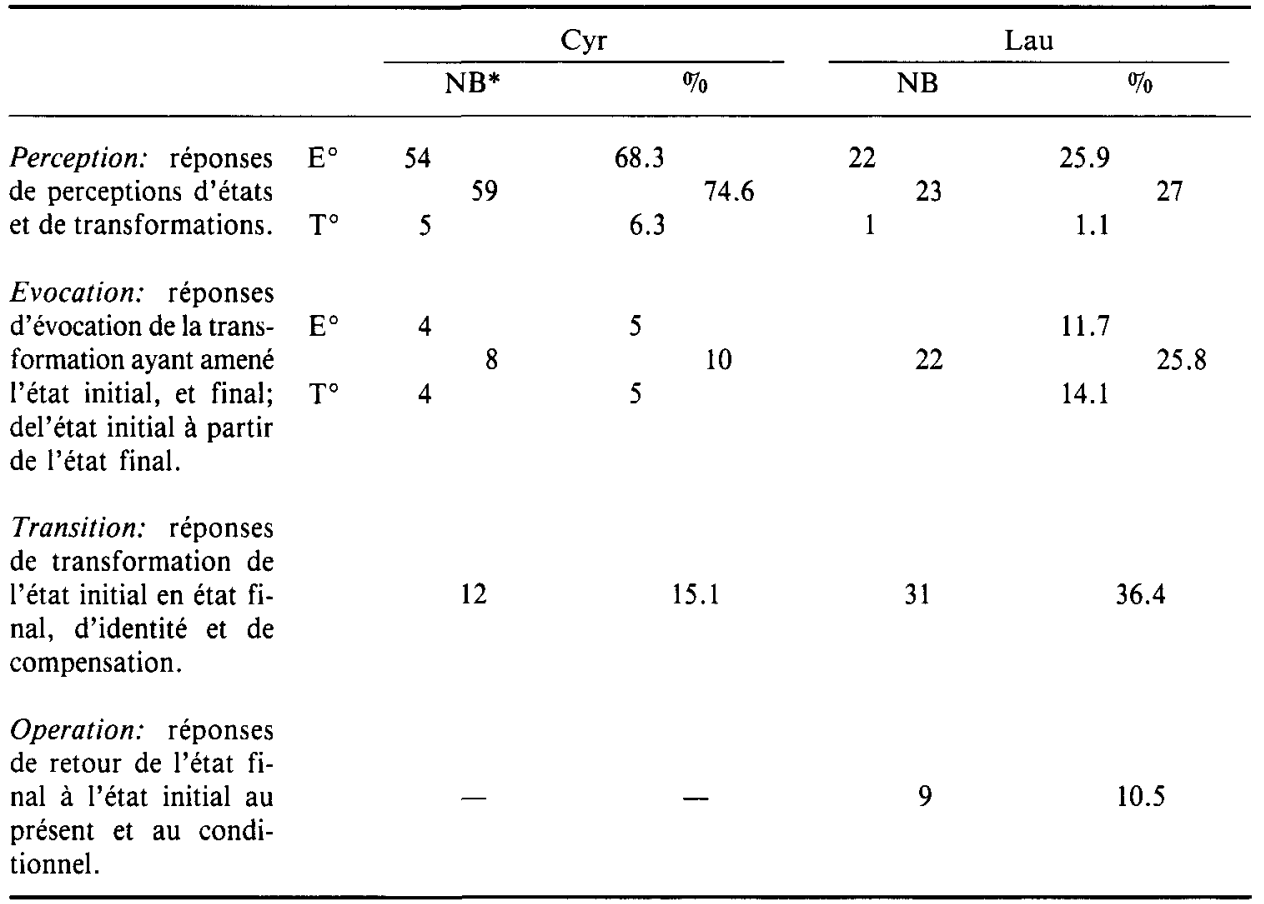

Clef: $\mathrm{NB}^{*}=$ nombre de réponses

$\mathrm{E}^{\circ}=$ états

$\mathrm{T}^{\circ}=$ transformations

\section{Degré d'autonomisation}

Nous obtenons ainsi pour chacun des enfants une valeur chiffrée du type d'argument le plus fourni, nous indiquant où il se situe dans sa genèse de l'activité de connaissance. Puis, pour chaque couple de jumeaux nous prenons la valeur la plus élevée, chez l'enfant le plus dépendant, à laquelle nous soustrayons la valeur correspondante du second. Le résultat ainsi obtenu sera une quantification du lien gémellaire, et donc par la même l'expression de l'autonomisation effective entre jumeaux.

$$
\text { ex: } 74.6-27=47.6
$$

La mise en parallèle de l'ensemble des résultats ainsi obtenus pour chaque couple de jumeaux nous a permis de construire une échelle relative au degré d'autonomisation, ou réciproquement à l'intensité du lien gémellaire. Nous avons des écarts entre les jumeaux d'un même couple qui s'échelonneraient de la façon suivante:

- le point 0 qui symbolise, de façon hypothétique, un lien inexistant, et donc une absence du couple gémellaire ayant laissé place à l'individu en tant que tel

- de 0 à 10 , les enfants son pratiquement autonomes, le lien gémellaire qui les unit est très faible 
— de 10 à 30 , l'autonomisation est toute relative et commence à être atteinte par leur caractéristique de jumeaux

- au delà de 30 , nous nous trouvons confronté à un développement cognitif en complet "parasitage"' d'un des enfants à l'égard de l'autre.

Cette échelle du lien unissant les jumeaux entre eux trouve son principe organisateur dans l'examen de leurs structures infra-logiques. En effet, ces aspects de la connaissance concernent les objets continus et permettent de structurer l'espace, le temps et la causalité. Ce sont eux qui vont être à l'origine de la capacité à entrer dans une pensée plus complexe. Les aspects logico-mathématiques, quant à eux vont concerner les objets discrets réunis en classes, sériés ou dénombrés, et donc dépassant leur simple place dans le réel pour devenir des éléments permettant à l'enfant d'organiser le monde qui l'entoure. Ces structures, plus élaborées, ne peuvent cependant être structurées sans le soutien de celles qui sont liées à la construction du réel.

Ainsi, si nous nous trouvons confronté à des enfants ayant peu d'autonomisation l'un à l'égard de l'autre, ou en d'autres termes face à un lien gémellaire très développé, l'enfant le plus dépendant de l'autre aura des structures sur le plan infra-logique très défaillantes et par la même des structures logico-mathématiques peu élaborées.

\section{CONCLUSIONS}

Ces défaillances structurales, si nous nous attachons à l'enfant en tant que tel qu'est le jumeau, se rapprochent fortement des troubles cognitifs qu'ont les enfants, en échec scolaire [8]. Ainsi, nous avons pu noter que pour les jumeaux qui avaient 8 ans et plus, systématiquement, les enseignants nous ont présenté l'un des deux comme ayant des difficultés scolaires. Suite à notre examen, celui-ci avait développé des procédures figuratives. Il nous apparaît que la procédure qui devrait nous permettre d'aboutir à une diminution de ce degré de gémellité, que nous parvenons à traduire en données chiffrées, serait la prise en charge des enfants dans le cadre d'une remédiation cognitive opératoire.

Ce mode d'intervention auprès des enfants a été élaboré au sein du laboratoire de psychologie génétique cognitive de terrain de l'Université Lumière, Lyon II [3].

Un tel mode de thérapie fait suite à un diagnostic opératoire ayant permis de découvrir la prédominance d'un mode de structuration figuratif de la connaissance chez un enfant. Il consiste à proposer au sujet des situations problèmes qui, à l'inverse de l'examen, se situent à un niveau juste suffisamment déséquilibrant pour pouvoir être compensé. Ces situations bien que nouvelles et donc perturbatrices de l'organisation interne du sujet ont cependant la particularité de permettre à des schèmes déjà construits de se mettre en activité, ce qui permet de susciter l'intérêt de l'enfant. Mais pour être résolues ces difficultés rencontrées nécessitent l'élaboration de nouvelles procédures. L'enfant parvient à faire de nouvelles découvertes qui progressivement vont se structurer et grâce à leur généralisation à différents contenus par feed-back positif vont donner naissance à de nouveaux schèmes, plus élaborés. La finalité de cette intervention est d'aider l'enfant à construire les éléments de connaissances qui lui font défaut par expérimentation (active et donc par abstraction pseudo-empirique), tout en l'amenant à expliciter ce qu'il fait. Ce qui lui permettra de construire le lien causal conscient. 
C'est de cette façon que l'enfant parviendra, progressivement, à générer ses propres perturbations et ses propres rééquilibrations, dans un système auto-constructeur et autorégulateur des connaissances (ce que Piaget a appelé l'équilibration majorante). C'est uniquement lorsqu'un tel mode de fonctionnement cognitif sera présent chez l'enfant que celui-ci parviendra à gérer la totale complexité du réel et à s'autonomiser.

Le mode d'intervention que nous allons mettre en place, et dont nous allons tenter de vérifier la pertinence, consistera:

- à faire passer un examen opératoire à chacun des enfants, dans un premier temps

- à les soumettre tous les deux à une remédiation cognitive opératoire.

En effet, même si nous trouvons un enfant ayant développé des structures plus aptes à gérer la complexité du réel que son conjoint, nous ne devons pas oublier que tous deux font partie intégrante de l'entité gémellaire. Ainsi, ils ont été affectés par cette situation, car ils ont un retard dans le développement de leurs structures cognitives, en comparaison à des enfants "uniques".

Notre travail consistera donc à permettre à l'enfant qui est le plus dépendant (ayant développé des procédures figuratives), et donc en potentialité d'échec scolaire, de développer des structures de la connaissance plus élaborées. Et parallèlement de travailler avec le second car nous sommes toujours dans une dynamique gémellaire. Précisons encore que toutes les variations sont possibles quant au mode d'intervention, mais que son organisation générale sera donnée par la mise à jour de l'intensité du lien unissant les jumeaux entre eux.

Nous sommes donc non seulement en mesure d'expliquer ce qu'est l'entité gémellaire et de quantifier sa prégnance, mais nous avons aussi la possibilité de prendre en considération les problèmes liés aux enfants dans leur individualité et de les traiter avec une très grande précision (quant au niveau où ils se situent dans leur structuration cognitive).

Compte tenu de ces éléments, nous pouvons élaborer un projet thérapeutique de remédiation cognitive opératoire, pour permettre au jumeau le plus dépendant d'avoir des structures cognitives plus efficientes et donc d'avoir plus d'emprise sur ce réel qui lui échappait jusqu'à présent.

En prenant en considération cette double dimension du couple et de l'individu nous avons la possiblité d'atténuer l'intensité du lien gémellaire et donc d'autonomiser ces enfants l'un par rapport à l'autre. Le déterminisme qui présidait à leur développement pourrait donc être effacé.

\section{BIBLIOGRAPHIE}

1. Allen MG, Pollin W, Hoffer A (1971): Parental birth and infancy factors in infant twin development. Psychiatry 127: 33-39.

2. Bak F (1994): Comprendre et résoudre les difficultés scolaires. Le Journal des psychologues 123: 38-41.

3. Bellano D (1992): De la genèse de l'organisation cognitive à la modélisation de l'activité de remédiation opératoire (du chaos vers l'ordre). Thèse de doctorat de Psychologie de l'Université Lumière Lyon II.

4. Bruggeman CC (1970): Twins: early grammatical development. Fol Phoniat 22: 197-215.

5. Cohen DJ, Dibble ED, Crawe JM (1977): Parental style: mothers' and fathers' perception of their relation with their children. Arch Gen Pyschiatry 34: 445-451. 
6. Conway D, Sauvé R (1977): The impact of twinship on parent child interaction. J Pers Soc Psychol 35: 97-107.

7. Dolle JM (1987): Au delà de Freud et Piaget (jalons pour de nouvelles perspectives en Psychologie). Toulouse: Privat.

8. Dolle JM, Bellano D (1989): Ces enfants qui n'apprennent pas (diagnostic et remédiations). Paris: Le centurion.

9. Goshen-Gottstein ER (1980): The mothering of twins, triplets and quadruplets. Psychiatry 43: 189-204.

10. Josse $D$, Robin M (1987): Deux bébés à la fois: une naissance bien singulière. In Passini (ed): L'après naissance en co-propriété. Genève: Médecine et hygiène.

11. Leonard ML (19617: Problems in identification and ego development in twins. Psychoanal Study Child 16: 300-320.

12. Minde KK, Perrota M, Corter C (1982): The effect of neonatal complications in same-sexed premature twins as their mother preference. J Am Acad Child Psychiatry 21: 446-452.

13. Mittler $P$ (1971): The study of twins London: Penguin.

14. Mittler $P$ (1976): Language development in young twins: biological, genetic and social aspects. Act Genet Med Gemellol 25: 359-365.

15. Piaget J (1938): La naissance de l'intelligence chez l'enfant. Neuchâtel: Delachaux et Niestlé.

16. Piaget $J$ (1938) La construction du réel chez l'enfant. Neuchâtel: Delachaux et Niestlé.

17. Piaget J, Inhelder B (1948): La représentation de l'espace chez l'enfant. Paris: P.U.F.

18. Piaget J, Inhelder B (1942): Le développement des quantités physiques chez l'enfant. Neuchâtel: Delachaux et Niestlé.

19. Piaget J (1958): La genèse des structures logiques élémentaires. Neuchâtel: Delachaux et Niestlé.

20. Piaget J, Szeminska A (1991): La genèse du nombre chez l'enfant. Neuchàtel: Delachaux et Niestlé.

21. Robin M, Josse D (1987): Quelques aspects de la relation mère-enfant à la suite d'une naissance gémellaire. Neuropsychiatrie de l'enfance et de l'adolescence 35: 369-377.

22. Robin M, Josse D, Tourette C (1988): Early mother twin interaction. Acta Genet Med Gemellol 37:151-159.

23. Sonnenschein S, Whitehurst G (1980): The development of communication when a bad model can be a good teacher. J Exp Child Psychol 30: 371-390.

24. Tourette C, Robin M, Josse D (1989): Treating twins as individuals: maternal educative practices. Eur J Psychol Educ 4; 2: 269-283.

25. Waterman $P$, Schatz $M(1982)$ : The acquisition of personal pronouns and proper names by an identical twin pair. JSHR 25:149-154.

26. Wilson RS (1974): Twins' mental development in the preschool years. Dev Psychol 10: 580-588.

27. Wilson RS (1975): Twins: patterns of cognitive development as measured on the Wechsler preschool and primary scale of intelligence. Dev Psychol 2: 126-134.

28. Wilson RS (1977): Twins and siblings: concordance for school-age mental development. Child Dev 48: 211-216.

29. Zazzo R (1986): Les jumeaux, le couple et la personne. Paris: P.U.F., p. 510.

30. Zazzo R (1990): Le paradoxe des jumeaux. Paris: Ed. Stock/Laurence Pernoud.

Correspondence: M. Fabrice Bak, 73 Rue Duquesne 69006 Lyon, France. 